\title{
Differential responses of canopy nutrients to experimental drought along a natural aridity gradient
}

\author{
Wentao Luo iD,${ }^{1}$ Xiaonn Zuo, ${ }^{2}$ Wang Ma, ${ }^{1}$ Chong Xu, ${ }^{3}$ Ang Li,${ }^{4}$ Qiang Yu, ${ }^{3}$ Alan K. Knapp, ${ }^{5}$ Roberto Tognetti, ${ }^{6,7}$ \\ Feike A. Dijkstra, ${ }^{8}$ Mai-He Li, ${ }^{1,9}$ Guodong Han, ${ }^{10}$ Zhengwen Wang, ${ }^{1,12}$ and XingGuo Han ${ }^{1,4,11}$ \\ ${ }^{1}$ Erguna Forest-Steppe Ecotone Research Station, Institute of Applied Ecology, Chinese Academy of Sciences, Shenyang 110164 China \\ ${ }^{2}$ Urat Desert-Grassland Research Station, Northwest Institute of Eco-Environment and Resources, Chinese Academy of Science, \\ Lanzhou 730000 China \\ ${ }^{3}$ National Hulunber Grassland Ecosystem Observation and Research Station, Institute of Agricultural Resources and Regional Planning, \\ Chinese Academy of Agricultural Sciences, Beijing 10008 China \\ ${ }^{4}$ State Key Laboratory of Vegetation and Environmental Change, Institute of Botany, Chinese Academy of Sciences, Beijing 100093 China \\ ${ }^{5}$ Graduate Degree Program in Ecology and Department of Biology, Colorado State University, Fort Collins, Colorado 80523 USA \\ ${ }^{6}$ Dipartimento di Agraria, Ambiente e Alimenti, Università del Molise, Campobasso 86090 Italy \\ ${ }^{7}$ European Forest Institute (EFI) Project Centre on Mountain Forests (MOUNTFOR), San Michele all'Adige 38010 Italy \\ ${ }^{8}$ Sydney Institute of Agriculture, School of Life and Environmental Sciences, The University of Sydney, Sydney, \\ New South Wales 2006 Australia \\ ${ }^{9}$ Forest Dynamics, Swiss Federal Institute for Forest, Snow and Landscape Research WSL, Birmensdorf CH-8903 Switzerland \\ ${ }^{10}$ College of Ecology and Environmental Science, Inner Mongolia Agricultural University, Hohhot 010018 China \\ ${ }^{11}$ University of Chinese Academy of Sciences, Beijing 100049 China
}

\begin{abstract}
The allocation and stoichiometry of plant nutrients in leaves reflect fundamental ecosystem processes, biotic interactions, and environmental drivers such as water availability. Climate change will lead to increases in drought severity and frequency, but how canopy nutrients will respond to drought, and how these responses may vary with community composition along aridity gradients is poorly understood. We experimentally addressed this issue by reducing precipitation amounts by $66 \%$ during two consecutive growing seasons at three sites located along a natural aridity gradient. This allowed us to assess drought effects on canopy nitrogen $(\mathrm{N})$ and phosphorus $(\mathrm{P})$ concentrations in arid and semiarid grasslands of northern China. Along the aridity gradient, canopy nutrient concentrations were positively related to aridity, with this pattern was driven primarily by species turnover (i.e., an increase in the relative biomass of $\mathrm{N}$ - and P-rich species with increasing aridity). In contrast, drought imposed experimentally increased $\mathrm{N}$ but decreased $\mathrm{P}$ concentrations in plant canopies. These changes were driven by the combined effects of species turnover and intraspecific variation in leaf nutrient concentrations. In addition, the sensitivity of canopy $\mathrm{N}$ and $\mathrm{P}$ concentrations to drought varied across the three sites. Canopy nutrient concentrations were less affected by drought at drier than wetter sites, because of the opposing effects of species turnover and intraspecific variation, as well as greater drought tolerance for nutrient-rich species. These contrasting effects of long-term aridity vs. short-term drought on canopy nutrient concentrations, as well as differing sensitivities among sites in the same grassland biome, highlight the challenge of predicting ecosystem responses to future climate change.
\end{abstract}

Key words: intraspecific variation; long-term aridity; manipulative experiment; nutritional response; sensitivity; short-term drought; species turnover.

\section{INTRODUCTION}

Droughts are projected to increase in magnitude and frequency in terrestrial ecosystems due to climate change (IPCC, 2013). Because nitrogen (N) and phosphorus (P) are essential nutrients for plant growth, maintenance, and reproduction (Reich and Oleksyn 2004, Li et al. 2017, Sardans et al. 2017), understanding the effects of increasing drought on the biogeochemistry of $\mathrm{N}$ and $\mathrm{P}$ is needed (Sardans and Peñuelas 2012). Patterns of plant nutrient allocation and stoichiometry reflect the use of $\mathrm{N}$ and $\mathrm{P}$ to sustain plant metabolism and growth (He et al. 2006, Yuan and Chen 2015). However, biogeochemical processes affecting $\mathrm{N}$ and $\mathrm{P}$ availability can be constrained by low soil water (Farooq et al. 2009), and in particular, the impact of drought on

Manuscript received 23 February 2018; revised 5 June 2018; accepted 18 June 2018. Corresponding Editor: Scott L. Collins.

${ }^{12}$ Corresponding Author. E-mail: wangzw@iae.ac.cn plant $\mathrm{N}$ and $\mathrm{P}$ concentrations (hereafter $[\mathrm{N}]$ and $[\mathrm{P}]$ ) remains unclear (Hartley et al. 2007, He and Dijkstra 2014).

During drought, low soil moisture and reduced microbial activity are known to retard decomposition and mineralization of organic matter, which in turn reduces the amount of $\mathrm{N}$ and $\mathrm{P}$ readily available for plant uptake (Hartley et al. 2007, He and Dijkstra 2014). Drought suppression of nutrient diffusion in soils, nutrient uptake by roots and nutrient transport in plant tissues have also been well-studied (Hartley et al. 2007, He and Dijkstra 2014). Plant N and P are tightly linked to many essential plant processes (e.g., photosynthesis, signal transduction, energy storage, Elser et al. 2007). However, the movement of $P$ in soils is more sensitive to drought stress than that of $\mathrm{N}$ (Lambers et al. 2008, Belnap 2011). Hence, shifts in plant $[N]$ and $[P]$ in response to drought stress may differ in magnitude and direction.

To date, much research has focused on assessing nutritional responses of individual species to drought, while such studies at the plant canopy scale are less common. However, 
shifts in nutrient dynamics in response to drought are more strongly relevant to ecosystem processes and functioning when assessed at the canopy level (Violle et al. 2012, Kichenin et al. 2013). Changes in the nutrient concentrations of plant canopies in response to drought can be due to species turnover, a result of species with different nutrient concentrations replacing others and a shift in relative abundance of each species, or intraspecific variation in nutrient concentrations arising from plasticity and genetic differentiation (Albert et al. 2010, Lepš et al. 2011, Violle et al. 2012).

Quantifying the relative contribution of species turnover and intraspecific variation is necessary to understand the responses of canopy nutrient concentrations to environmental change. Short-term droughts, for example, may induce canopy functional responses mainly through shifts in the relative biomass of each species and intraspecific variation, while longer-term shifts in water availability may impact canopies primarily through species turnover (Lajoie and Vellend 2015, Volf et al. 2016). When changing in the same direction, shifts in species' relative biomass and intraspecific variation in nutrient concentration can magnify canopy nutrient responses to environmental changes, whereas opposing changes can weaken responses.

Patterns of sensitivity to water limitation and drought can be estimated by investigating the responses of canopy nutrients to changes in water availability along aridity gradients (Körner 1989, Dunne et al. 2003, Luo et al. 2015). Indeed, the degree of species turnover observed along such gradients may be an important factor influencing ecosystem resilience as aridity increases (Dunne et al. 2003, 2004, Sandel et al. 2010). At shorter time scales, precipitation change can be experimentally imposed and responses in the nutrient dynamics of plant canopies evaluated (Körner 1989, Dunne et al. 2003, He and Dijkstra 2014, Luo et al. 2015). Intraspecific responses are more likely to play a role in the resistance and stability of ecosystems to short-term drought (Dunne et al. 2004, Sandel et al. 2010).

Conducting field drought experiments at multiple sites across environmental gradients (e.g., precipitation) within a biome can provide unique insights (Dunne et al. 2004, Peñuelas et al. 2004, Sandel et al. 2010, Wolkovich et al., 2012, Yuan et al. 2017). For example, several recent studies have concluded that responses of plant communities in the same biome can vary dramatically with such variation (Gilgen and Buchmann 2009, Cherwin and Knapp 2012, Knapp et al. 2015, Zefferman et al. 2015), often linked to differences in productivity and resource availability (Grime et al. 2000, Ochoa-Hueso et al. 2018). While observed ecological responses to drought typically include reductions in net primary productivity and species richness, altered carbon cycle processes and in some cases, extensive mortality (Hoover et al. 2018), few studies have explored the responses of canopy nutrient concentrations to reduced water availability. Multi-site drought experiments represent one approach to more comprehensively understand the underlying biogeochemical mechanisms of the vegetation response to climate change (Kröel-Dulay et al. 2015, Hoover et al. 2018).

We conducted a standardized climate manipulative experiment across three sites that span an aridity gradient in arid and semiarid grasslands of northern China to assess responses of canopy nutrients to short-term drought vs. long-term water limitations. We selected sites with a uniform geomorphic template, distinct precipitation amounts, similar temperatures, and low human disturbance, to provide an ideal platform for assessing drought sensitivity across this water- and nutrient-limited biome. We hypothesized that plant canopy nutrient concentrations would respond differently to short-term drought vs. long-term water stress (assessed via the aridity gradient). Reductions in canopy nutrient concentrations caused by natural aridity were expected to mainly result from species turnover, while those caused by short-term drought would be from intraspecific variation. We also predicted that the sensitivity of canopy nutrient concentrations to short-term drought would vary across sites, increasing from the least to the most arid site.

\section{Material And Methods}

\section{Study sites and experimental design}

In 2014 (pretreatment year), three sites with a relatively narrow range of mean annual temperature but significantly different mean annual precipitation were established in arid and semiarid grasslands of northern China (Fig. 1). The eastern site (low aridity, aridity index $[\mathrm{AI}]=0.40$ ) with a mean annual precipitation of $346 \mathrm{~mm}$ was located at the Inner Mongolia Grassland Ecosystem Research Station $\left(116^{\circ} 33^{\prime} \mathrm{E}, 43^{\circ} 32^{\prime} \mathrm{N}\right.$; Bai et al. 2004). The central site

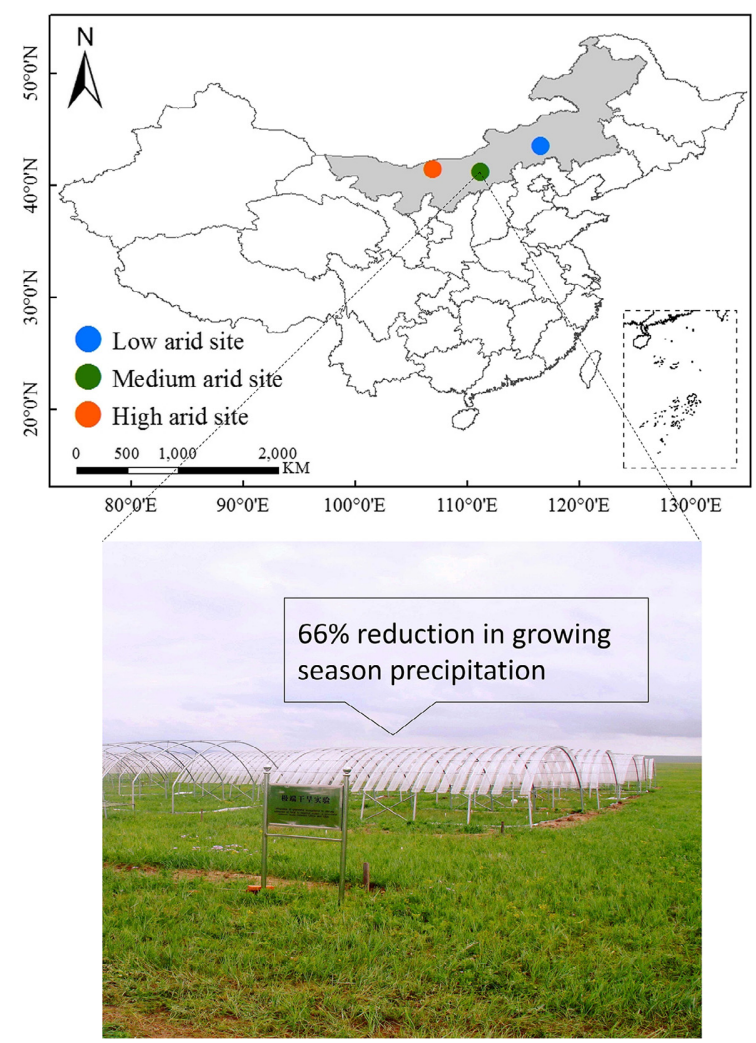

FIG. 1. Map of the locations of study sites arranged along a natural aridity gradient in the arid and semiarid grasslands of northern China. At each site, a manipulative drought experiment (photo) was conducted using rainout shelters to impose a $66 \%$ reduction in growing season precipitation. See text for further details. 
(medium aridity, $\mathrm{AI}=0.25)$ with a mean annual precipitation of $251 \mathrm{~mm}$ was located in Sheila MuRen $\left(111^{\circ} 53^{\prime} \mathrm{E}\right.$, $41^{\circ} 47^{\prime} \mathrm{N}$ ) and the western site (high aridity, $\left.\mathrm{AI}=0.17\right)$ with a mean annual precipitation of $175 \mathrm{~mm}$ was located at the Urat Desert-Grassland Research Station (106 $58^{\prime} \mathrm{E}, 41^{\circ} 25^{\prime}$ $\mathrm{N}$; Appendix S1: Fig. S1). More information about the dynamics and variation of precipitation and temperature for the three sites is shown in Appendix S1: Fig. S1. Mean annual temperature ranged from $1.9^{\circ} \mathrm{C}$ in the east to $5.9^{\circ} \mathrm{C}$ in the west along this aridity gradient. Grassland communities at all sites were dominated by perennial species $(90 \%$ of the total biomass) with plant cover and productivity decreasing with increasing aridity. The dominant species were Stipa grandis and Leymus chinensis in the low aridity site, S. grandis and Stellera chamaejasme in the medium aridity site, and Stipa breviflora and Peganum harmala in the high aridity site. Soils in this region are predominantly chestnut soils rich in calcium, equivalent to Calcicorthic Aridisols according to the US system of soil taxonomy.

At each site, we imposed a $66 \%$ reduction in ambient growing season precipitation from May to August in 2015 and 2016 (Fig. 1), which was about $50 \%$ of annual precipitation in those years. The experimental design was a randomized complete block design with six replications in a relatively flat area with plant communities representative of the larger area. Each $6 \times 6 \mathrm{~m}$ plot was hydrologically isolated by trenching the perimeter to a depth of $1 \mathrm{~m}$; the trench was lined with 6-mm-thick plastic and metal flashing to prevent lateral subsurface and surface water flow into plots. In the center of each plot, $16 \mathrm{~m}^{2}(4 \times 4 \mathrm{~m})$ sampling plots were established. The drought-treatment plots were covered by a light scaffolding that supported strips of transparent polyethylene (Beijing Plastics Research Institute, Beijing, China). The clear strips were arranged at a density sufficient to passively reduce each rainfall event by $\sim 66 \%$ during the growing season. The roof was $2 \mathrm{~m}$ above ground at the highest point and the rain shelters did not extend to ground level, allowing for near surface air exchange and avoiding unwanted greenhouse effects. This rainout shelter design was developed by Yahdjian and Sala (2002) and has been used extensively in a wide range of ecosystems globally, due to its low cost and minimal influence on the microclimate (Yahdjian and Sala 2002, Wilcox et al. 2015). Untreated control plots were also trenched, but lacked roof scaffolding. We assessed shelter effects on the light environment by measuring transmittance of photosynthetically active radiation under full sun conditions. The effects of the shelter on the light environment were small, permitting nearly $90 \%$ transmission, which most likely did not influence ecosystem functions, because illumination is not a limiting factor for plant growth in this region.

Soil temperature and moisture content were measured with a soil temperature/moisture sensor (PG-110; Jingchuang Electronic Technology Co., Handan, China) placed at $10-\mathrm{cm}$ depth in the center of each sampling plot. Air temperature and relative humidity were measured with an air temperature/humidity sensor (PG-310; Jingchuang Electronic Technology Co.) mounted at $10-\mathrm{cm}$ above the ground in the center of each sampling plot. Measurements were recorded on a data logger every hour and averaged to produce daily mean values.

\section{Sampling and analysis}

In August 2014 (pretreatment year), a quadrat $(2 \times 2 \mathrm{~m})$ was established in each sampling plot and divided into four sub-quadrats $(1 \times 1 \mathrm{~m})$. All species present in each subquadrat were recorded. Percent cover of each species was estimated using a $1 \times 1 \mathrm{~m}$ metal frame divided into 100 equal grids. The number of grid junctions whose vertical projections overlapped with each plant was recorded.

In August 2016, after 2 yr of imposed drought, aboveground biomass of each species was harvested by clipping at ground level and plant foliage was collected from the most abundant species. These abundant species were defined as those that collectively comprised at least $90 \%$ of total biomass in the plot. Foliage was collected from three individuals of each species per plot. The youngest, fully expanded leaves of each individual were sampled for nutrient analysis. Plant material was oven-dried at $80^{\circ} \mathrm{C}$ to constant weight in the laboratory.

After removing the litter layer, five soil cores $(0-10 \mathrm{~cm}$ depth) were collected from each sampling plot, using a soil corer (2.5-cm diameter). Soil samples were homogenized by hand mixing, and separated into two sub-samples: one was stored at $4{ }^{\circ} \mathrm{C}$ immediately after collection for later measurement of initial gravimetric moisture content and soil available $[\mathrm{N}]\left(\mathrm{NH}_{4}{ }^{+}-\mathrm{N}\right.$ plus $\left.\mathrm{NO}_{3}{ }^{-}-\mathrm{N}\right)$; the other sample was air dried and stored at room temperature.

Fresh soils were passed through a $2-\mathrm{mm}$ sieve, and roots and rocks were removed. Gravimetric moisture content was measured by drying soil subsamples at $105^{\circ} \mathrm{C}$ for $48 \mathrm{~h}$. Additional fresh soil samples were extracted with $50 \mathrm{~mL}$ of $2 \mathrm{~mol} / \mathrm{L} \mathrm{KCl}$, and the filtered soil extract was used to determine soil available [N] with a continuous flow spectrophotometer (FIAstar 5000, Foss Tecator, Hillerød, Denmark).

Air-dried soils were crushed and passed through a 2-mm sieve. Available [P] was determined by extracting the soil with $0.5 \mathrm{~mol} / \mathrm{L} \mathrm{NaHCO}_{3}$ at $\mathrm{pH} 8.5$ and analyzed using the molybdenum blue-ascorbic acid method (Olsen 1954). Additionally, dried plant and soil samples were ground to pass through a $1-\mathrm{mm}$ sieve. Plant $[\mathrm{P}]$ was measured by inductively coupled plasma emission spectroscopy (OPTIMA 3000 DV; Perkin Elmerr, Boston, MA, USA) after $\mathrm{H}_{2} \mathrm{SO}_{4}-\mathrm{H}_{2} \mathrm{O}_{2}$ digestion (Kuo 1996). Total [N] of plants and soils was measured using an elemental analyzer (2400II CHN elemental analyzer; Perkin-Elmer) with a combustion temperature of $950^{\circ} \mathrm{C}$ and a reduction temperature of $640^{\circ} \mathrm{C}$.

\section{Data analysis}

For plant data recorded in August of 2014 before the drought treatment started, species richness $(S)$ was calculated as the number of species in each plot, while ShannonWeiner diversity index $(H)$ and Pielou evenness index $(E)$ were calculated using $H=-\sum_{i=1}^{S} P_{i} \times \ln P_{i}$ and $E=\left(-\sum_{i=1}^{S} P_{i} \times \ln P_{i}\right) / \ln S$, respectively, in each plot at each site. Where $P_{i}$ is the relative coverage of species $i$ and $S$ is the species richness in each plot.

Plant canopy nutrient concentrations were defined as overall mean nutrient concentrations across all species weighted by each species' relative biomass in each plot (Kichenin et al. 2013). As changes in canopy nutrient 
concentrations can be attributed to both species turnover and intraspecific variation, their relative contributions were separated using the following equation (Jung et al. 2014): $C_{\text {Turn }}=N_{\text {Dr* }}-N_{\mathrm{Ct}}$ and $C_{\text {Intra }}=N_{\mathrm{Dr}}-N_{\mathrm{Dr}}$. Here, $N_{\mathrm{Ct}}$ and $N_{\mathrm{Dr}}$ are canopy mean nutrient concentrations in the control and drought plots, respectively, calculated from relative biomass and nutrient concentrations of each species measured in their respective plot. $N_{\text {Dr* }}$ is the canopy nutrient concentrations in the drought-treated plots, recalculated using species' relative biomass in the drought-treated plots, but the nutrient concentrations measured in the control plots. $C_{\text {Turn }}$ and $C_{\text {Intra }}$ represent the isolated effects of species turnover and intraspecific variation, respectively, in driving the canopy nutritional responses to drought manipulation.

Using the above-mentioned methods we then calculated the contributions of species turnover and intraspecific variation in canopy mean nutrient concentrations along the aridity gradient (Kichenin et al. 2013). We recalculated $N_{\text {Dr* }}$ from the relative biomass of each species in the control plots at each site, but now using the mean species nutrient concentrations in the control plots across all three sites.

Plant canopy $[\mathrm{N}]$ and $[\mathrm{P}]$, soil total $[\mathrm{N}]$ and available $[\mathrm{N}]$ and $[\mathrm{P}]$, as well as species richness, diversity and evenness were analyzed using a mixed-model analysis of variance with drought treatment and site as fixed factors and block as a random factor. When interactive effects of drought treatment and site were significant, the mixed model analysis of variance was separately applied for each site with drought treatment as fixed factor and block as random factor. Student's $T$ test was performed to test the significance of the effects of species turnover (i.e., $N_{\mathrm{Dr} *}$ vs. $N_{\mathrm{Ct}}$ ) and intraspecific variation (i.e., $N_{\mathrm{Dr} *}$ vs. $N_{\mathrm{Dr}}$ ) within each site.

Relationships between canopy and soil nutrient concentrations and between canopy nutrient concentrations and soil moisture were examined with a linear mixed model incorporating random effects for each site and among the three sites along the natural aridity gradient. Blocks were used as a random factor within each site, while blocks nested within site were used as the random factor among the three sites along the aridity gradient.

For each of the six blocks at each site, plant canopy nutrient concentrations in the control plots were paired with those in the drought-treated plots, resulting in 36 data pairs. Sensitivity was estimated by the relative change in the drought-treated plots compared with the control plots as follows: Sensitivity $=\left(N_{\mathrm{Dr}}-N_{\mathrm{Ct}}\right) / N_{\mathrm{Ct}}$ (Hsu et al. 2012, Zhang et al. 2017). Sensitivity indicates a relative change in canopy nutrient concentration $(\%)$ induced by the same relative reduction (\%) in precipitation at each site. The sensitivities at the three sites were compared using one-way ANOVAs, with Duncan's Test as the post-hoc test for multiple comparisons.

Before statistical analysis, Shapiro-Wilk and Levene's Tests were used to test the normality and heteroscedasticity of all data, respectively. Due to their normal distribution and homogeneity of variance, original data were used in our statistical analysis without log-transformation. All statistical analyses were carried out using the SPSS 13.0 for Windows ${ }^{\circledR}$ statistics program (SPSS Inc., Chicago, Illinois, USA, 2004) and the nlme package in $\mathrm{R}$ ( $\mathrm{R}$ i386 3.1.1).

\section{RESULTS}

During the 2015 and 2016 growing seasons, the drought treatment reduced precipitation below the 25 th percentile of historical precipitation probability distribution functions based on long-term records of growing season precipitation for each site (Appendix S1: Fig. S2). In contrast, growing season precipitation was normal in both years (approx. 50th percentile of historic amounts) for the control treatments (Appendix S1: Fig. S2).

Prior to imposing experimental drought, species richness, diversity and evenness were similar between control and treatment plots at each site (Appendix S1: Fig. S3). However, along the aridity gradient, species shifted to those with higher $[\mathrm{N}]$ and $[\mathrm{P}]$ from the least to the most arid site (Appendix S1: Fig. S4). Accordingly, plant canopy $[\mathrm{N}]$ and $[\mathrm{P}]$ also increased progressively from the least to the most arid site $(P<0.05$, Fig. 2). Effects of species turnover on canopy $[\mathrm{N}]$ and $[\mathrm{P}]$ were significantly higher in the high than the low aridity site $(P<0.05)$, while intraspecific effects were very weak and did not change along the aridity gradient (Fig. 2). Therefore, shifts in plant canopy $[\mathrm{N}]$ and $[\mathrm{P}]$ were primarily caused by species turnover, which explained more than $90 \%$ of the variance.

As expected, soil moisture content was greatly reduced by the imposed drought but effects on soil and air temperature, and relative humidity were minimal (Appendix S1: Fig. S5). Two new species appeared within the plant community at the low aridity site; the other species at this site had altered relative biomass, increased average foliar $[\mathrm{N}]$ and reduced average foliar $[\mathrm{P}]$ in drought vs. control plots (Appendix S1: Table S1 and Fig. S4). At the medium aridity site, two species with relatively low $[\mathrm{N}]$ and $[\mathrm{P}]$ disappeared within the plant community under drought conditions, while relative biomass and average foliar $[\mathrm{N}]$ and $[\mathrm{P}]$ were altered in the remaining species (Appendix S1: Table S1 and Fig. S4). Finally, within the plant community at the high aridity site, one disappeared, two increased their relative biomass and one reduced its relative biomass in response to drought (Appendix S1: Table S1 and Fig. S4). Mean foliar [N] and $[\mathrm{P}]$ were generally higher in the control than drought-treated plots at this site (Appendix S1: Table S1 and Fig. S4).

The treatment $\times$ site interaction was significant for $[\mathrm{N}]$ $(P<0.05)$ and marginally significant for $[\mathrm{P}](P<0.1)$, indicating that the response to drought of plant canopy $[\mathrm{N}]$ and $[\mathrm{P}]$ varied across sites (Table 1). Drought increased plant canopy $[\mathrm{N}]$ at each site, but this was significant only at the medium aridity site $(P<0.05$, Fig. 3$)$; canopy $[\mathrm{P}]$ was reduced at each site, but only at the low aridity site was this response significant $(P<0.05$, Fig. 3). Changes in plant canopy $[\mathrm{N}]$ and $[\mathrm{P}]$ caused by drought were due to both intraspecific variation $(28-74 \%)$ and species turnover (26-72\%; Fig. 3). The effects of species turnover and intraspecific variation were synergistic for plant canopy $[\mathrm{N}]$ at the medium aridity site and for plant canopy $[\mathrm{P}]$ at the low aridity site (Fig. 3). In contrast, the effects were antagonistic for canopy $[\mathrm{N}]$ at both low and high aridity sites and for canopy $[\mathrm{P}]$ at the medium and high aridity sites (Fig. 3). For plant canopy [N], intraspecific variation contributed more at the low and medium aridity sites, while species turnover contributed more at the high aridity site (Fig. 3). Contributions of intraspecific variation were 


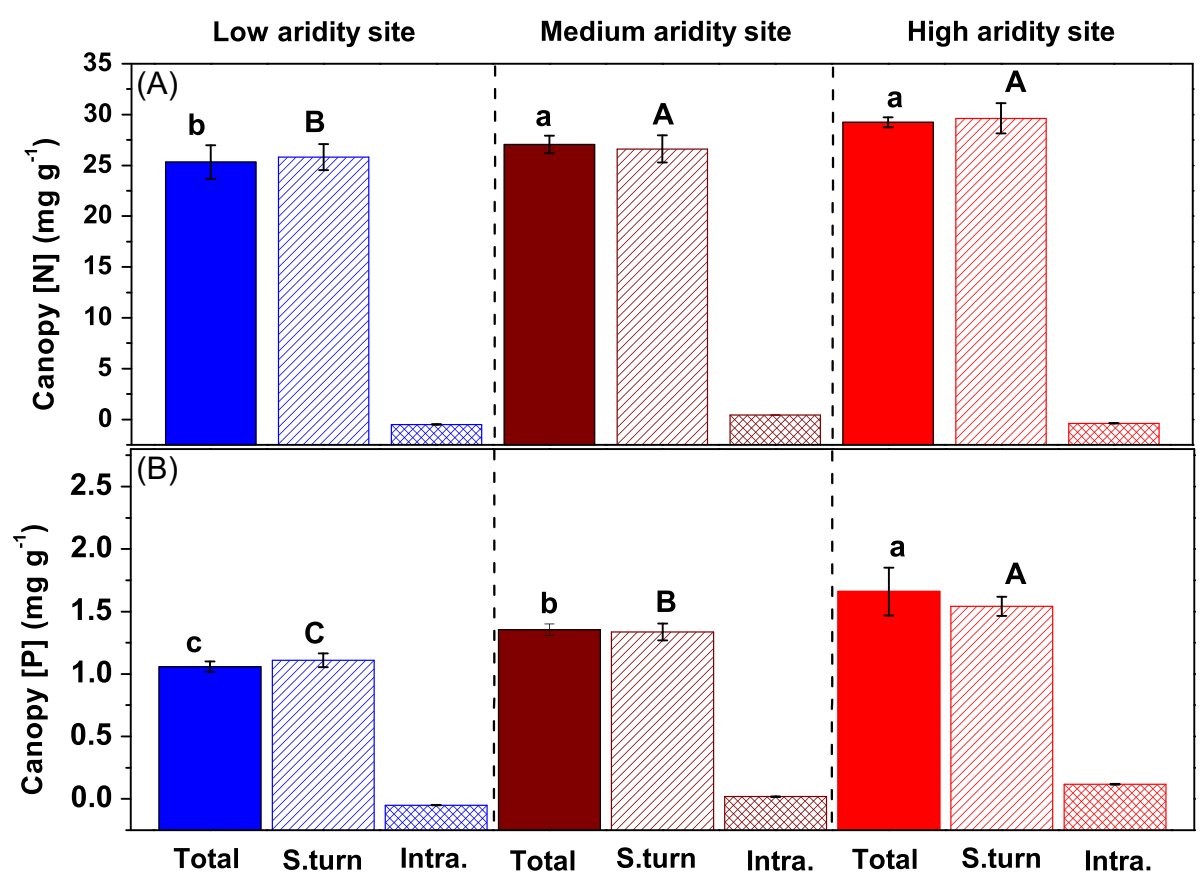

FIG. 2. Values of canopy $[\mathrm{N}]$ and $[\mathrm{P}]$ measured at three sites along a natural gradient in arid and semiarid grasslands of northern China. The figure presents variations in canopy $[\mathrm{N}]$ and $[\mathrm{P}]$ due to both species turnover and intraspecific variation (total variation), species turnover only and intraspecific variation only along the aridity gradient. Total variation corresponds to the observed canopy $[\mathrm{N}]$ and $[\mathrm{P}]$ in the control plots of each site; species turnover corresponds to the canopy $[\mathrm{N}]$ and $[\mathrm{P}]$ recalculated using the relative biomass of each species at each site but mean $[\mathrm{N}]$ and $[\mathrm{P}]$ across all three sites; intraspecific variation corresponds to the difference between the above two measured values (total variation - species turnover). Nutrient concentrations are shown as mean $\pm \mathrm{SE}(n=6)$. Lowercase and capital letters indicate significant differences $(P<0.05)$ in total variation and species turnover among sites, respectively.

TABLE 1. Results of mixed model analysis of variance for plant canopy $[\mathrm{N}]$ and $[\mathrm{P}]$ and soil total $[\mathrm{N}]$, available $[\mathrm{N}]$ and $[\mathrm{P}]$. Drought treatment (drought vs. control) and site (low, medium and high aridity sites) were used as fixed factors and block as a random factor.

\begin{tabular}{|c|c|c|c|c|c|}
\hline & Canopy $[\mathrm{N}]$ & Canopy $[\mathrm{P}]$ & Total $[\mathrm{N}]$ & Available [N] & Available $[\mathrm{P}]$ \\
\hline Treatment & 0.005 & 0.047 & 0.101 & 0.515 & 0.002 \\
\hline Site & 0.017 & $<0.001$ & $<0.001$ & 0.273 & 0.001 \\
\hline Treatment $\times$ Site & 0.043 & 0.087 & 0.061 & 0.961 & 0.669 \\
\hline
\end{tabular}

Note: Significant $P$ values $(P \leq 0.05)$ and marginally significant values $(0.1 \geq P>0.05)$ are given in bold and italics, respectively; $n=6$.

significant at the low and medium aridity sites $(P<0.05)$, while contributions of species turnover were significant at the high aridity site $(P<0.01$, Fig. 3). For plant canopy $[\mathrm{P}]$, intraspecific variation contributed more at the high aridity site, while species turnover contributed more at the low and medium aridity sites (Fig. 3). Contributions of intraspecific variation were marginally significant at the high aridity site $(P<0.1)$, while contributions of species turnover were significant at the medium aridity site $(P<0.01)$ and marginally significant at the high aridity site $(P<0.1$, Fig. 3$)$.

The treatment $\times$ site interaction was marginally significant for soil total $[\mathrm{N}](P<0.1)$, but non-significant for soil available $[\mathrm{N}]$ and $[\mathrm{P}]$ (Table 1 ). Plant canopy $[\mathrm{N}]$ was negatively correlated with soil total $[\mathrm{N}]$ at the medium aridity site $(P<0.05)$, while canopy $[\mathrm{P}]$ was negatively correlated with soil available $[\mathrm{P}]$ at the low aridity site $(P<0.05$, Appendix S1: Fig. S6). Along the aridity gradient, plant canopy $[\mathrm{N}]$ decreased with an increase in soil total $[\mathrm{N}](P<0.01)$, while canopy $[\mathrm{P}]$ decreased with an increase in available $[\mathrm{P}](P<0.05$, Appendix S1: Fig. S6). Plant canopy $[\mathrm{N}]$ was marginally negatively correlated with soil moisture content at each site $(P<0.1)$, whereas canopy $[\mathrm{P}]$ was positively correlated with soil moisture content at the low $(P<0.05)$ and medium $(P<0.1)$ aridity sites where the latter was marginal (Fig. 4). Plant canopy $[N]$ and $[\mathrm{P}]$ significantly decreased with an increase in soil moisture along the aridity gradient $(P<0.05$, Fig. 4$)$.

Sensitivities of plant canopy $[\mathrm{N}]$ and $[\mathrm{P}]$ responses to imposed drought varied across the three sites (Fig. 5). The medium aridity site was the most sensitive to drought for plant canopy $[\mathrm{N}]$ while the low aridity site was the most sensitive for plant canopy $[\mathrm{P}](P<0.05$, Fig. 5$)$. The high aridity site was the least sensitive to drought for both plant canopy $[\mathrm{N}]$ and $[\mathrm{P}](P<0.05$, Fig. 5).

\section{DisCUSSION}

\section{Different responses to short-term drought and long-term aridity}

Along the aridity gradient, plant canopy nutrient concentrations increased with increasing aridity, primarily from the much larger effects of species turnover in comparison with 


\section{Canopy N}
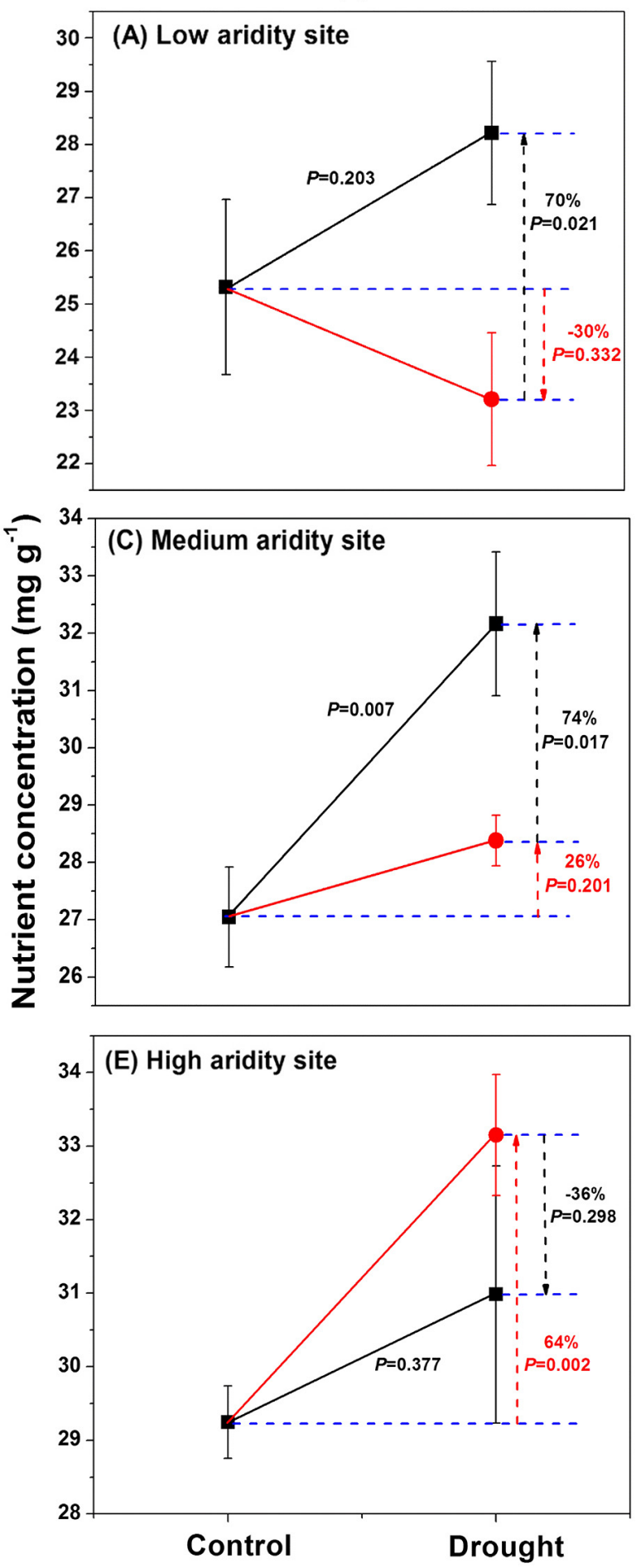

\section{Canopy P}
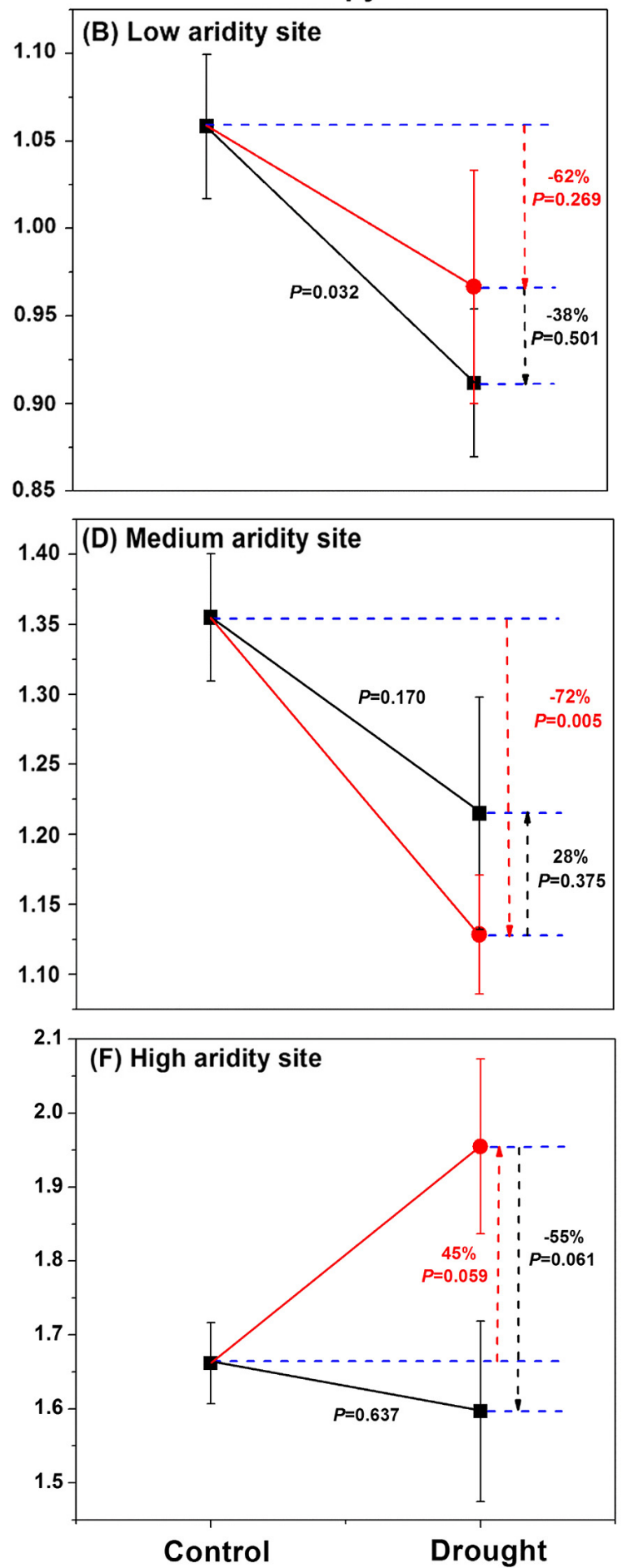

FIG. 3. Changes in canopy $[\mathrm{N}]$ and $[\mathrm{P}]$ in response to drought in arid and semiarid grasslands of northern China. This figure shows the response estimated to be due to both species turnover and intraspecific variation and due to species turnover only for each site. A and B represent the low aridity site; $\mathrm{C}$ and $\mathrm{D}$ represent the medium aridity site; $\mathrm{E}$ and $\mathrm{F}$ represent the high aridity site. Black squares correspond to canopy $[\mathrm{N}]$ and $[\mathrm{P}]$ in the control and drought plots; Red circles correspond to canopy $[\mathrm{N}]$ and $[\mathrm{P}]$ in the drought plots recalculated from species $[\mathrm{N}]$ and $[\mathrm{P}]$ in the control plots. Arrows indicate the contributions of species turnover (red arrows) and of intraspecific variation (black arrows) to the change in canopy nutrient concentrations. Species turnover and intraspecific variation are expressed as percentages of their cumulative magnitude. A parallel direction of shift in species turnover and intraspecific variation effects indicates positive (synergistic) covariation, whereas an opposite direction of shift in species turnover and intraspecific variation indicates negative (antagonistic) covariation. Nutrient concentrations are shown as mean $\pm \mathrm{SE}(n=6)$. Values of $P$ are shown. 


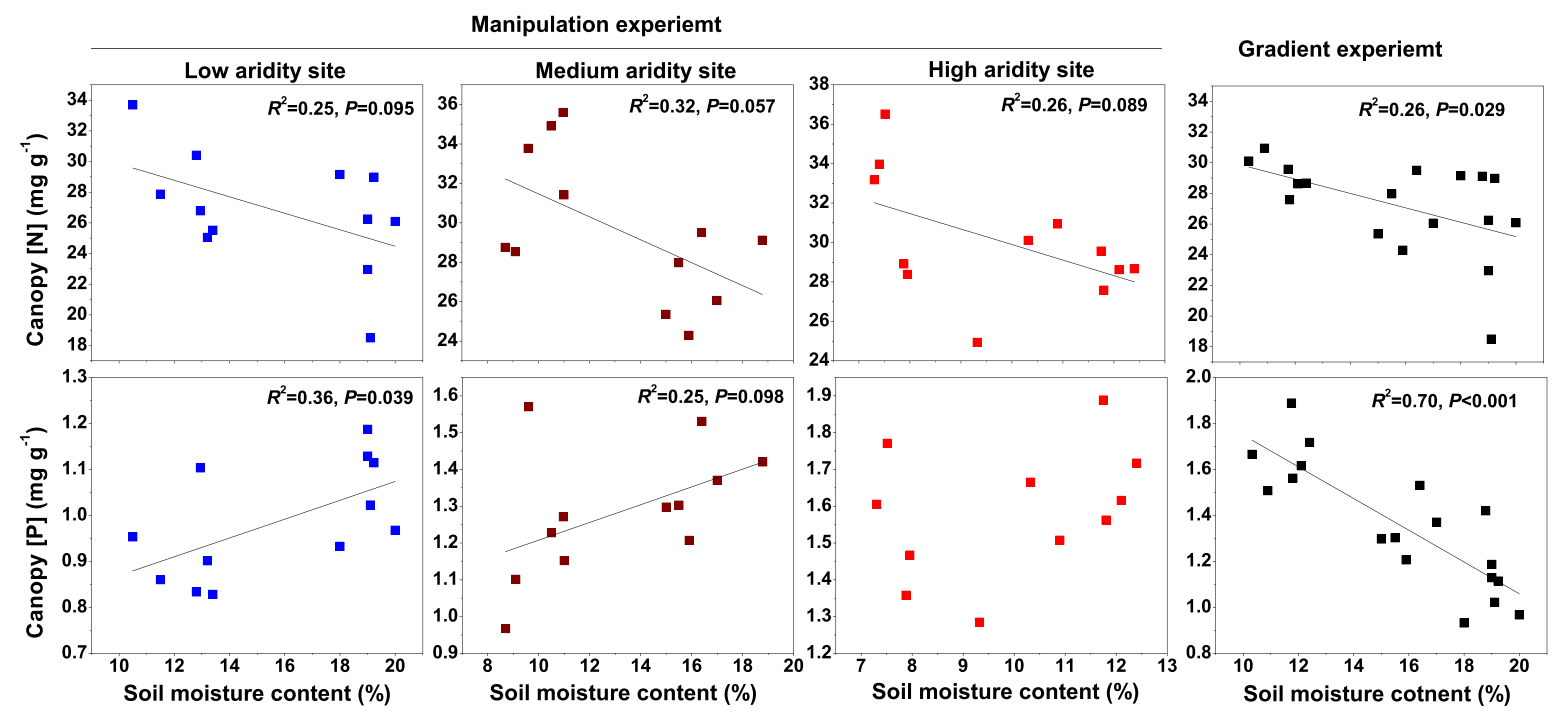

FIG. 4. Relationships between canopy $[\mathrm{N}]$ and $[\mathrm{P}]$ and soil moisture content $(0-10 \mathrm{~cm})$ under drought conditions within and among three grassland sites along an aridity gradient in northern China. Values of $R^{2}$ and $P$ are shown for significant $(P<0.05)$ and marginal significant $(P<0.10)$ regressions. Soil moisture content $(0-10 \mathrm{~cm})$ is the mean value for the May to August growing season.
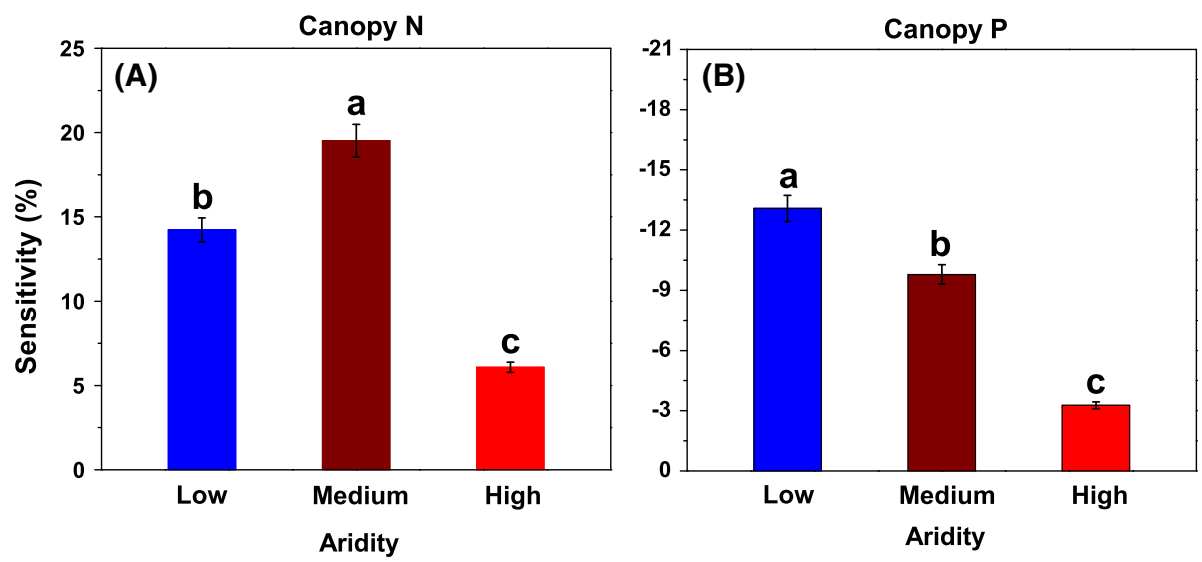

FIG. 5. Sensitivity of canopy $[\mathrm{N}]$ and $[\mathrm{P}]$ to drought for each site within arid and semiarid grasslands of northern China. Columns with different lowercase letters are significantly different $(P<0.05)$ according to Duncan's Test.

intraspecific variation (Fig. 2). Overall, there was a gradual replacement of species by those with relatively higher nutrient concentrations with increasing aridity (Appendix S1: Fig. S4). These results indicate that long-term reductions in precipitation can lead to an increase in species with higher nutrient concentrations. Our findings are consistent with studies reporting higher plant nutrient concentrations in arid vs. semiarid ecosystems in northern China (Liu et al. 2010, Luo et al. 2015) and elsewhere (Wright and Westoby 2002, Wright et al. 2003, Sandel et al. 2010). The shift in the plant community towards species with higher nutrient concentrations may provide a competitive advantage for species under water stress, especially when water stress persists or becomes more severe (Waraich et al. 2011, Weih et al. 2011, Sardans and Peñuelas 2012, Li et al. 2017). Plant nutrient concentrations are positively correlated with leaf area-based photosynthetic rates in arid and semiarid grassland plants (Wright and Westoby 2002, Liu et al. 2010), and thus higher nutrient concentrations in arid regions can help plants opportunistically maximize photosynthesis during periods when water is available, enhancing their competitiveness in harsh environments (Farquhar et al. 2002, Weih et al. 2011).

In contrast to these responses to the aridity gradient, canopy $[\mathrm{N}]$ and $[\mathrm{P}]$ responses to short-term drought imposed experimentally were more variable, with canopy nutrients altered by both intraspecific variation and species turnover (Fig. 3). Thus, these two approaches (experimental vs. gradient) identified key differences in plant community responses to drought at short- and long-term scales (Wilcox et al. 2016). Manipulative experiments reveal short-term changes in community structure in response to drought (Sandel et al. 2010, Smith et al. 2015, Wilcox et al. 2016, Yuan et al. 2017). In this case, plant community composition usually remains relatively constant, with variability in canopy nutrient concentration mostly explained by responses of the extant species to reductions in precipitation (Smith et al. 2009, Lajoie and Vellend 2015). However, the expectation is that initial ecosystem responses to drought 
will differ from longer-term responses, perhaps due to the delayed responses of the plant community to long-term resource feedbacks, growth, and competition (Dunne et al. 2004, Peñuelas et al. 2004, Smith et al. 2009, 2015, Sandel et al. 2010, Wilcox et al. 2016). In this case, both species turnover and intraspecific variation may contribute to the drought effects on plant canopy nutrient concentrations in both magnitude and direction (Fig. 3). However, the response of plant canopy $[\mathrm{N}]$ and $[\mathrm{P}]$ concentrations to longterm drought will be much larger when the plant community composition changes substantially (Smith et al. 2009).

Plant nutrient concentrations typically reflect soil nutrient availability (biogeochemical hypotheses, Reich and Oleksyn 2004). Thus, we predicted that the availability of soil nutrients may be altered by aridity/drought, altering nutrients supplied to plants. This could also lead to variation in plant nutrient concentrations. However, both the aridity gradient and the drought experiment failed to show such a relationship between plant and soil nutrient concentrations (Appendix S1: Fig. S6). Previous studies have also reported a mismatch between plant nutrient status and soil nutrient supply (Luo et al. 2015, Riva et al. 2015). Such a mismatch between nutrients in the soil and plants may be may be caused by severely low soil water content, which can limit nutrient mass flow within soils and also the nutrient acquisition by plant roots (Khasanova et al. 2013, He and Dijkstra 2014). This interpretation is supported by the correlations between canopy nutrient concentrations and soil water availability in our study (Fig. 4). Moreover, the lack of concordance between soil and canopy nutrient concentration may also reflect the difficulty in characterizing season-long nutrient availability from one-time sampling of soils (Reichmann et al. 2013).

\section{Different sensitivity to drought manipulation among sites}

Responses in species turnover and intraspecific variation of canopy nutrients to short-term drought were at times synergistic, and at other times antagonistic (i.e., positive and negative covariation, Fig. 3). Interestingly, the responses of nutrient concentrations to drought stress were significant when the effects of species turnover and intraspecific variation on nutrients were synergistic but not antagonistic (Fig. 3). Other studies have shown that significant reductions in plant canopy [N] (Jung et al. 2014) and [P] (Kichenin et al. 2013) mediated by species turnover could be dampened by the effect of intraspecific variation. These results indicate that both species turnover and intraspecific variation can play a key role in driving the net responses of canopy nutrients to changes in precipitation.

Differential sensitivities of plant canopy nutrient concentrations to drought were evident across the three sites (Fig. 5). Similar results have been reported for plant productivity (Heisler-White et al. 2009, Byrne et al. 2013, Wilcox et al. 2015), $\mathrm{CO}_{2}$ flux (Hoover et al. 2014) and plant species richness (Cleland et al. 2013). The sensitivity of plant canopy nutrient concentration to drought was highest for $\mathrm{N}$ at the medium aridity site and for $\mathrm{P}$ at the low aridity site (Fig. 5). This result was consistent with the positive covariation observed between species turnover and intraspecific variation (Fig. 3). For example, two species (A. umbellata and C. squarrosa) with relatively low foliar $[\mathrm{N}]$ disappeared, while all other species increased their average foliar $[\mathrm{N}]$ and relative biomass in response to drought at the medium aridity site (Appendix S1: Table S1 and Fig. S4). Positive covariation implies that drought selected for species with increased nutrient concentrations and a greater potential to increase foliar nutrients (Jung et al. 2014).

Consistent with our second hypothesis, canopy nutrient concentrations at the site with the lowest precipitation were least sensitivity to drought (Fig. 5), driven by equal but opposite effects of among- vs. within-species variation (Fig. 3). Increasing drought at the high aridity site favored the species with relatively higher nutrient concentrations. Thus, $A$. fruticulosa (relatively low nutrient concentrations) was lost from this community, whereas $A$. mongolicum and $P$. harmala (relatively high nutrient concentrations) increased their relative biomass with drought (Appendix S1: Table S1 and Fig. S4). However, short-term drought at the high aridity site caused an intraspecific decrease in canopy nutrient concentrations (Fig. 3). Mean values of nutrient concentrations for all three species decreased due to drought (Appendix S1: Table S1 and Fig. S4). Together, these results suggest that species with higher nutrient concentrations have competitive advantages and may eventually displace extant individuals with reduced foliar nutrient concentrations with long-term drought, consistent with patterns quantified along the aridity gradient.

\section{Conclusions}

We investigated both short- and long-term effects of drought on plant canopy nutrient concentrations, using identical drought experiments at three sites along a natural aridity gradient. We found that plant canopy $[\mathrm{N}]$ and $[\mathrm{P}]$ increased with increasing aridity along the aridity gradient, while canopy $[\mathrm{N}]$ increased but $[\mathrm{P}]$ decreased in response to short-term (2 yr) drought. Changes in canopy nutrient concentrations caused by long-term aridity mainly resulted from species turnover, while those caused by short-term drought resulted from both species turnover and intraspecific nutrient variability. The sensitivity of canopy nutrient concentrations to drought also varied across sites, with the most arid sites being the least sensitive. Our findings demonstrate that responses of canopy nutrient concentrations to drought can differ substantially between experimental and gradient approaches, as well as among different sites within a single biome. This implies that integrating experiments into gradient studies (Beier et al. 2012) provides a more comprehensive perspective for understanding and predicting biogeochemical responses of ecosystems to drought.

\section{ACKNOWLedgments}

We thank all who worked in the Extreme Drought in Grasslands Experiment (EDGE) project for assistance in planning and conducting the field experiment. Qiang Yu is a co-corresponding author for this manuscript. He designed the experiment, and all questions or correspondence about the experiment should be forwarded to him (yuqiang@caas.cn). In addition, we are grateful to Professor Chengjie Wang in Inner Mongolia Agricultural University for providing long-term climate data. We thank two anonymous referees as well as the Handling Editor for constructive comments on the manuscript. This study was supported by funding from Natural Science Foundation of China (41320104002, 41600302 and 41622103) and National Key Research and Development Program of China (2016YFC0500601 and 2016YFC0500602). 


\section{Literature Cited}

Albert, C. H., W. Thuiller, N. G. Yoccoz, R. Douzet, S. Aubert, and S. Lavorel. 2010. A multi-trait approach reveals the structure and the relative importance of intra- $v s$. interspecific variability in plant traits. Functional Ecology 24:1192-1201.

Bai, Y., X. Han, J. Wu, Z. Chen, and L. Li. 2004. Ecosystem stability and compensatory effects in the Inner Mongolia grassland. Nature 431:181-184.

Beier, C., et al. 2012. Precipitation manipulation experiments-challenges and recommendations for the future. Ecology Letters 15:899-911.

Belnap, J. 2011. Biological phosphorus cycling in dryland regions. Pages 371-406 in E. K. Bunemann, A. Oberson, and E. Frossard, editors. Phosphorus in action. Springer, Berlin, Heidelberg, Germany.

Byrne, K. M., W. K. Lauenroth, and P. B. Adler. 2013. Contrasting effects of precipitation manipulations on production in two sites within the central grassland region, USA. Ecosystems 16:10391051.

Cherwin, K., and A. Knapp. 2012. Unexpected patterns of sensitivity to drought in three semi-arid grasslands. Oecologia 169:845-852.

Cleland, E. E., et al. 2013. Sensitivity of grassland plant community composition to spatial vs. temporal variation in precipitation. Ecology 94:1687-1696.

Dunne, J. A., J. Harte, and K. J. Taylor. 2003. Subalpine meadow flowering phenology responses to climate change: integrating experimental and gradient methods. Ecological Monographs 73:69-86.

Dunne, J. A., S. R. Saleska, M. L. Fischer, and J. Harte. 2004. Integrating experimental and gradient methods in ecological climate change research. Ecology 85:904-916.

Elser, J. J., M. E. Bracken, E. E. Cleland, D. S. Gruner, W. S. Harpole, H. Hillebrand, J. T. Ngai, E. W. Seabloom, J. B. Shurin, and J. E. Smith. 2007. Global analysis of nitrogen and phosphorus limitation of primary producers in freshwater, marine and terrestrial ecosystems. Ecology Letters 10:1135-1142.

Farooq, M., A. Wahid, N. Kobayashi, D. Fujita and S. Basra. 2009. Plant drought stress: effects, mechanisms and management. Pages 153-188 in E. Lichtfouse, M. Navarrete, P. Debaeke, S. Véronique, and C. Alberola, editors. Sustainable agriculture. Springer, Dordrecht, The Netherlands.

Farquhar, G. D., T. N. Buckley, and J. M. Miller. 2002. Optimal stomatal control in relation to leaf area and nitrogen content. Silva Fennica 36:625-637.

Gilgen, A. K., and N. Buchmann. 2009. Response of temperate grasslands at different altitudes to simulated summer drought differed but scaled with annual precipitation. Biogeosciences 6:2525-2539.

Grime, J. P., V. K. Brown, K. Thompson, G. J. Masters, S. H. Hillier, I. P. Clarke, A. P. Askew, D. Corker, and J. P. Kielty. 2000. The response of two contrasting limestone grasslands to simulated climate change. Science 289:762-765.

Hartley, I. P., A. Heinemeyer, and P. Ineson. 2007. Effects of three years of soil warming and shading on the rate of soil respiration: substrate availability and not thermal acclimation mediates observed response. Global Change Biology 13:1761-1770.

He, M., and F. A. Dijkstra. 2014. Drought effect on plant nitrogen and phosphorus: a meta-analysis. New Phytologist 204:924-931.

He, J. S., J. Fang, Z. Wang, D. Guo, D. F. B. Flynn, and Z. Geng. 2006. Stoichiometry and large-scale patterns of leaf carbon and nitrogen in the grassland biomes of China. Oecologia 149:115-122.

Heisler-White, J. L., J. M. Blair, E. F. Kelly, K. Harmoney, and A. K. Knapp. 2009. Contingent productivity responses to more extreme rainfall regimes across a grassland biome. Global Change Biology 15:2894-2904.

Hoover, D. L., A. K. Knapp, and M. D. Smith. 2014. Contrasting sensitivities of two dominant $\mathrm{C}_{4}$ grasses to heat waves and drought. Plant Ecology 215:721-731.
Hoover, D. L., K. R. Wilcox, and K. E. Young. 2018. Experimental droughts with rainout shelters: a methodological review. Ecosphere 9:e02088.

Hsu, J. S., J. Powell, and P. B. Adler. 2012. Sensitivity of mean annual primary production to precipitation. Global Change Biology 18:2246-2255.

IPCC. 2013. Climate change. The physical and science basis. Summary for the policymakers, technical summary and frequently asked questions. Part of the I Contribution to the Fifth Assessment Report of the Intergovernmental Panel on Climate Change. IPCC, Geneva, Switzerland.

Jung, V., C. H. Albert, C. Violle, G. Kunstler, G. Loucougaray, and T. Spiegelberger. 2014. Intraspecific trait variability mediates the response of subalpine grassland communities to extreme drought events. Journal of Ecology 102:45-53.

Khasanova, A., J. J. James, and R. E. Drenovsky. 2013. Impacts of drought on plant water relations and nitrogen nutrition in dryland perennial grasses. Plant and Soil 372:541-552.

Kichenin, E., D. A. Wardle, D. A. Peltzer, C. W. Morse, and G. T. Freschet. 2013. Contrasting effects of plant inter- and intraspecific variation on community-level trait measures along an environmental gradient. Functional Ecology 27:1254-1261.

Knapp, A. K., C. J. W. Carroll, E. M. Denton, K. J. La Pierre, S. L. Collins, and M. D. Smith. 2015. Differential sensitivity to regionalscale drought in six central US grasslands. Oecologia 177:949-957.

Körner, C. 1989. The nutritional status of plants from high altitudes. Oecologia 81:379-391.

Kröel-Dulay, G., et al. 2015. Increased sensitivity to climate change in disturbed ecosystems. Nature Communications 6:6682.

Kuo, S. 1996. Phosphorus. Pages 869-920 in D. L. Sparks, A. L. Page, P. A. Loeppert, et al., editors. Methods of soil analysis part 3: chemical methods. Soil Science Society of America and American Society of Agronomy, Madison, Wisconsin, USA.

Lajoie, G., and M. Vellend. 2015. Understanding context dependence in the contribution of intraspecific variation to community trait-environment matching. Ecology 96:2912-2922.

Lambers, H., F. S. Chapin, and T. L. Pons. 2008. Plant physiological ecology. Springer, New York, New York, USA.

Lepš, J., F. De Bello, P. Smilauer, and J. Dolezal. 2011. Community trait response to environment: disentangling species turnover $v s$. intraspecific trait variability effects. Ecography 34:856-863.

Li, F., et al. 2017. Warming effects on permafrost ecosystem carbon fluxes associated with plant nutrients. Ecology 98:2851-2859.

Liu, G. F., G. T. Freschet, X. Pan, J. H. C. Cornelissen, Y. Li, and M. Dong. 2010. Coordinated variation in leaf and root traits across multiple spatial scales in Chinese semi-arid and arid ecosystems. New Phytologist 188:543-553.

Luo, W. T., et al. 2015. Plant nutrients do not covary with soil nutrients under changing climatic conditions. Global Biogeochemical Cycles 29:1298-1308.

Ochoa-Hueso, R., S. L. Collins, M. Delgado-Baquerizo, K. Hamonts, W. T. Pockman, R. L. Sinsabaugh, M. D. Smith, A. K. Knapp, and S. A. Power. 2018. Drought consistently alters the composition of soil fungal and bacterial communities in grasslands from two continents. Global Change Biololgy 24:28182827.

Olsen, S. R. 1954. Estimation of available phosphorus in soils by extraction with sodium bicarbonate. United States Department of Agriculture Circular 939, United States Government Printing Office, Washington, D.C., USA.

Peñuelas, J., C. Gordon, L. Llorens, T. Nielsen, A. Tietema, C. Beier, P. Bruna, B. Emmett, M. Estiarte, and A. Gorissen. 2004. Nonintrusive field experiments show different plant responses to warming and drought among sites, seasons, and species in a north-south European gradient. Ecosystems 7:598-612.

Reich, P. B., and J. Oleksyn. 2004. Global patterns of plant leaf N and $\mathrm{P}$ in relation to temperature and latitude. Proceedings of the National Academy of Sciences USA 101:11001-11006. 
Reichmann, L. G., O. E. Sala, and D. P. Peters. 2013. Water controls on nitrogen transformations and stocks in an arid ecosystem. Ecosphere 4:11.

Riva, E. G., I. M. Pérez-Ramos, A. Tosto, C. M. Navarro-Fernández, M. Olmo, T. Marañón, and R. Villar. 2015. Disentangling the relative importance of species occurrence, abundance and intraspecific variability in community assembly: a trait-based approach at the whole-plant level in Mediterranean forests. Oikos 125:354-363.

Sandel, B., L. J. Goldstein, N. J. Kraft, J. G. Okie, M. I. Shuldman, D. D. Ackerly, E. E. Cleland, and K. N. Suding. 2010. Contrasting trait responses in plant communities to experimental and geographic variation in precipitation. New Phytologist 188:565575 .

Sardans, J., M. Bartrons, O. Margalef, A. Gargallo-Garriga, I. A. Janssens, P. Ciais, M. Obersteiner, B. D. Sigurdsson, H. Y. Chen, and J. Peñuelas. 2017. Plant invasion is associated with higher plant-soil nutrient concentrations in nutrient-poor environments. Global Change Biology 23:1282-1291

Sardans, J., and J. Peñuelas. 2012. The role of plants in the effects of global change on nutrient availability and stoichiometry in the plant-soil system. Plant Physiology 160:1741-1761.

Smith, M. D., A. K. Knapp, and S. L. Collins. 2009. A framework for assessing ecosystem dynamics in response to chronic resource alterations induced by global change. Ecology 90:3279-3289.

Smith, M. D., et al. 2015. Global environmental change and the nature of aboveground net primary productivity responses: insights from long-term experiments. Oecologia 177:935-947.

Violle, C., B. J. Enquist, B. J. Mcgill, L. Jiang, C. H. Albert, C. Hulshof, V. Jung, and J. Messier. 2012. The return of the variance: intraspecific variability in community ecology. Trends in Ecology \& Evolution 27:244-252.

Volf, M., et al. 2016. Effects of long- and short-term management on the functional structure of meadows through species turnover and intraspecific trait variability. Oecologia 180:941-950.

Waraich, E. A., R. Ahmad, Saifullah, M. Y. Ashraf, and Ehsanullah. 2011. Role of mineral nutrition in alleviation of drought stress in plants. Australian Journal of Crop Science 5:764-777.
Weih, M., L. Bonosi, L. Ghelardini, and A. C. Ronnberg-Wastljung. 2011. Optimizing nitrogen economy under drought: Increased leaf nitrogen is an acclimation to water stress in willow (Salix spp.). Annals of Botany 108:1347-1353.

Wilcox, K. R., J. C. Von Fischer, J. M. Muscha, M. K. Petersen, and A. K. Knapp. 2015. Contrasting above- and belowground sensitivity of three Great Plains grasslands to altered rainfall regimes. Global Change Biology 21:335-344.

Wilcox, K. R., J. M. Blair, M. D. Smith, and A. K. Knapp. 2016. Does ecosystem sensitivity to precipitation at the site-level conform to regional-scale predictions? Ecology 97:561-568.

Wolkovich, E. M., et al. 2012. Warming experiments underpredict plant phenological responses to climate change. Nature 485:494 497.

Wright, I. J., P. B. Reich, and M. Westoby. 2003. Least-cost input mixtures of water and nitrogen for photosynthesis. American Naturalist 161:98-111.

Wright, I. J., and M. Westoby. 2002. Leaves at low versus high rainfall: coordination of structure, lifespan and physiology. New Phytologist 155:403-416.

Yahdjian, L., and O. E. Sala. 2002. A rainout shelter design for intercepting different amounts of rainfall. Oecologia 133:95-101.

Yuan, Z. Y., and H. Y. H. Chen. 2015. Decoupling of nitrogen and phosphorus in terrestrial plants associated with global changes. Nature Climate Change 5:465-469.

Yuan, Z. Y., F. Jiao, X. R. Shi, J. Sardans, F. T. Maestre, M. Delgado-Baquerizo, P. B. Reich, and J. Peñuelas. 2017. Experimental and observational studies find contrasting responses of soil nutrients to climate change. elife $6: \mathrm{e} 23255$.

Zefferman, E., J. T. Stevens, G. K. Charles, M. Dunbar-Irwin, T. Emam, S. Fick, L. V. Morales, K. M. Wolf, D. J. Young, and T. P. Young. 2015. Plant communities in harsh sites are less invaded: a summary of observations and proposed explanations. AoB Plants, 7:plv056.

Zhang, B., X. Tan, S. Wang, M. Chen, S. Chen, T. Ren, J. Xia, Y. Bai, J. Huang, and X. Han. 2017. Asymmetric sensitivity of ecosystem carbon and water processes in response to precipitation change in a semi-arid steppe. Functional Ecology, 31:1301-1311.

\section{SUPPORTING INFORMATION}

Additional supporting information may be found in the online version of this article at http://onlinelibrary.wiley.com/doi/10.1002/ecy. 2444/suppinfo 